\title{
TITLE:
}

\section{Transverse Acoustic Excitations in Liquid $\mathrm{Ga}$}

\section{$\operatorname{AUTHOR}(\mathrm{S}):$}

Hosokawa, S.; Inui, M.; Kajihara, Y.; Matsuda, K.; Ichitsubo, T.; Pilgrim, W. -C.; Sinn, H.; ... Gonzalez, D. J.; Tsutsui, S.; Baron, A. Q. R.

\section{CITATION:}

Hosokawa, S. ... [et al]. Transverse Acoustic Excitations in Liquid Ga. Physical Review Letters 2009, 102(10): 105502.

\section{ISSUE DATE:}

2009-03

URL:

http://hdl.handle.net/2433/109872

\section{RIGHT:}

(c) 2009 The American Physical Society 


\title{
Transverse Acoustic Excitations in Liquid Ga
}

\author{
S. Hosokawa, ${ }^{1,2, *}$ M. Inui, ${ }^{3}$ Y. Kajihara,${ }^{3}$ K. Matsuda, ${ }^{4}$ T. Ichitsubo, ${ }^{4}$ W.-C. Pilgrim, ${ }^{2}$ H. Sinn, ${ }^{5}$ L. E. González, ${ }^{6}$ \\ D. J. González, ${ }^{6}$ S. Tsutsui, ${ }^{7}$ and A. Q. R. Baron ${ }^{7,8}$ \\ ${ }^{1}$ Center for Materials Research Using Third-Generation Synchrotron Radiation Facilities, Hiroshima Institute of Technology, \\ Hiroshima 731-5193, Japan \\ ${ }^{2}$ Physikalische Chemie, Fachbereich Chemie, Philipps Universiät Marburg, 35032 Marburg, Germany \\ ${ }^{3}$ Graduate School of Integrated Arts and Sciences, Hiroshima University, Higashi-Hiroshima 739-8521, Japan \\ ${ }^{4}$ Department of Materials Science and Engineering, Graduate School of Engineering, Kyoto University, Kyoto 606-8501, Japan \\ ${ }^{5}$ HASYLAB, DESY, 22607 Hamburg, Germany \\ ${ }^{6}$ Departamento de Física Teórica, Universidad de Valladolid, 47011 Valladolid, Spain \\ ${ }^{7}$ SPring-8/JASRI, Hyogo 679-5198, Japan \\ ${ }^{8}$ SPring-8/RIKEN, Hyogo 679-5148, Japan \\ (Received 20 November 2008; published 13 March 2009)
}

\begin{abstract}
The transverse acoustic excitation modes were detected by inelastic x-ray scattering in liquid Ga in the $Q$ range above $9 \mathrm{~nm}^{-1}$ although liquid $\mathrm{Ga}$ is mostly described by a hard-sphere liquid. An ab initio molecular dynamics simulation clearly supports this finding. From the detailed analysis for the $S(Q, \omega)$ spectra with a good statistic quality, the lifetime of $0.5 \mathrm{ps}$ and the propagating length of $0.4-0.5 \mathrm{~nm}$ can be estimated for the transverse acoustic phonon modes, which may correspond to the lifetime and size of cages formed instantaneously in liquid Ga.
\end{abstract}

DOI: 10.1103/PhysRevLett.102.105502

PACS numbers: 63.50.-x, 61.20.Ne, 61.25.Mv

Lattice modes, or collective modes, are modes of vibration of the entire systems. In the long wavelength limit, they may be described by the classical theory of elasticity in which the system is treated as a continuum possessing macroscopic elastic constants. As the wavelength gets shorter, approaching the distance between atoms, the microscopic structure of the system and the forces between individual pairs of atoms become the dominant features in determining the nature of the modes of vibration.

In simple liquids, longitudinal acoustic phonon modes are always observed by ultrasonic, optical, or inelastic scattering experiments because the density fluctuations can occur due to a strong repulsive force between the atoms when they approach each other. However, transverse phonon modes usually cannot be detected in liquids by ultrasonic or optical measurements because the shear force in the long spatial range is very weak if the liquid has no longrange network such as covalent glass-forming materials like $\mathrm{SiO}_{2}$ [1].

When the atomic vibration wavelength in a liquid approaches the atomic nearest neighbor distance, i.e., in the terahertz frequency region, the situation changes. There may be a solidlike cage effect on the nanometer scale that acts as a restoring force for acoustic transverse modes. If the above speculation is correct, transverse acoustic modes could be realized experimentally even in liquid metals in dynamic structure factor $S(Q, \omega)$ measurements by inelastic x-ray or neutron scattering (IXS or INS) which covers the terahertz-nanometer range.

The transverse acoustic modes in simple liquids have been discussed theoretically for more than 30 years.
Levesque and co-workers [2] performed a molecular dynamic (MD) simulation using a Lenard-Jones potential, which revealed the existence of the transverse acoustic modes in simple liquids. Hansen et al. [3] presented a MD work of time-dependent correlation functions of a classical one-component plasma and pointed out the existence of high-frequency transverse modes for large $Q$ values. This has been reviewed in several textbooks on simple liquids [4-6]. Shimojo et al. [7] and Kahl and Kambayashi [8] performed MD simulations using more realistic potentials for liquid $\mathrm{Na}$ and $\mathrm{Rb}$, respectively. However, the contribution of the transverse acoustic modes to $S(Q, \omega)$ was negligible since their magnitudes are relatively small and they are highly damped. Thus, the transverse modes in liquid alkali metals may have been masked by the strong and broad longitudinal acoustic excitations and/or quasielastic peaks.

In a previous IXS study on liquid Ga, narrow longitudinal acoustic phonon modes were observed, indicating a positive deviation from the hydrodynamic velocity of sound by about $13 \%$ [9]. In addition, subpicosecond correlations between the neighboring atoms or short-living covalent bonds were found in the feature of quasielastic lines at the first structure factor $S(Q)$ maximum [10]. Moreover, transverselike low-energy excitations were observed as shoulders of the quasielastic peak [10]. This finding may be related to the appearance of the short-living covalent bonds. However, the fit results were scattered as a function of $Q$ due to the insufficient statistics, and it was impossible to discuss the dynamical properties of the transverselike excitations in detail. In order to confirm the 
existence of the transverse acoustic modes and to obtain transverse elastic properties, we have precisely measured IXS on liquid $\mathrm{Ga}$ at $40{ }^{\circ} \mathrm{C}$ at the beam line BL35XU with an excellent energy resolution of about $1.5 \mathrm{meV}$ and intense $\mathrm{x}$-ray photon flux of about $4 \times 10^{9}$ photons $^{-1}$. Details of the IXS spectrometer were given elsewhere [11]. The $Q$ resolution was set to be $\pm 0.5 \mathrm{~nm}^{-1}$.

The Ga sample was contained in a thin-walled $(0.25 \mathrm{~mm})$ single-crystal sapphire cell, which is the socalled Tamura-type cell [12]. The high x-ray absorption of liquid $\mathrm{Ga}$ was handled by reducing the sample thickness to about $50 \mu \mathrm{m}$. The cell was placed in a vessel [13] equipped with single-crystal $\mathrm{Si}$ thin windows capable of covering the scattering angles between $0^{\circ}$ and $25^{\circ}$. It was applied with 1.5 bar of high purity grade $\mathrm{He}$ gas. The temperature of $40^{\circ} \mathrm{C}$ was achieved by using a Mo resistant heater and monitored and controlled with two $\mathrm{W}-5 \% \mathrm{Re}-\mathrm{W}-26 \% \operatorname{Re}$ thermocouples. The liquid phase of the sample was confirmed by measuring $S(Q, 0)$ spectra during the experiment.

Figure 1 shows the results of $S(Q, \omega) / S(Q)$ at $Q=$ $6.7-10.6 \mathrm{~nm}^{-1}$ together with the resolution function shown by the dashed curve at the bottom of the figure. At these $Q$ values, longitudinal acoustic excitations are clearly seen as peaks at about $12-18 \mathrm{meV}$. Besides the longitudinal excitations, small but clear extra excitations can be observed at $Q=9.2$ and $10.6 \mathrm{~nm}^{-1}$ as shoulders between the quasielastic peak and longitudinal acoustic excitation peaks at about 5-8 meV. With decreasing $Q$, these extra modes become invisible below $Q=7.9 \mathrm{~nm}^{-1}$.

The $S(Q, \omega)$ data were analyzed by using a damped harmonic oscillator (DHO) model [14] expressed as

$$
\left[\frac{1}{1-e^{-\hbar \omega / k_{B} T}}\right] \frac{A_{Q}}{\pi} \frac{4 \omega \omega_{Q} \Gamma_{Q}}{\left(\omega^{2}-\omega_{Q}^{2}\right)^{2}+4 \Gamma_{Q}^{2} \omega^{2}}
$$

for two excitation modes each and a Lorentzian for the quasielastic line. Here $A_{Q}$ and $\omega_{Q}$ are the amplitude and energy of the inelastic excitation modes, respectively, and $\Gamma_{Q}$ is the width close to half width at half maximum.

The solid curves in Fig. 1 indicate the best fits and residuals (multiplied by two) of the single and double DHO (1DHO and 2DHO, respectively) models, convolved with the resolution function, to the experimental data. At $Q=10.6 \mathrm{~nm}^{-1}$, the residual for the $1 \mathrm{DHO}$ model has large minima at about $\pm 3 \mathrm{meV}$ as indicated by arrows and broad maxima in the larger $\omega$ range up to $\sim 10 \mathrm{meV}$, which means that the experimental quasielastic peak is narrower than the $1 \mathrm{DHO}$ model and an extra excitation mode is necessary at 5-8 meV. At $Q=9.2 \mathrm{~nm}^{-1}$, the $1 \mathrm{DHO}$ model is still insufficient as shown in the residuals, although the inconsistency of the $1 \mathrm{DHO}$ fit becomes small.

The $2 \mathrm{DHO}$ model reproduces well the experimental $S(Q, \omega)$ data beyond $Q \sim 9 \mathrm{~nm}^{-1}$. The dotted curve is a Lorentzian for the quasielastic peak, and the dashed and chain curves are, respectively, the DHO functions for the longitudinal and transverselike inelastic modes. As shown

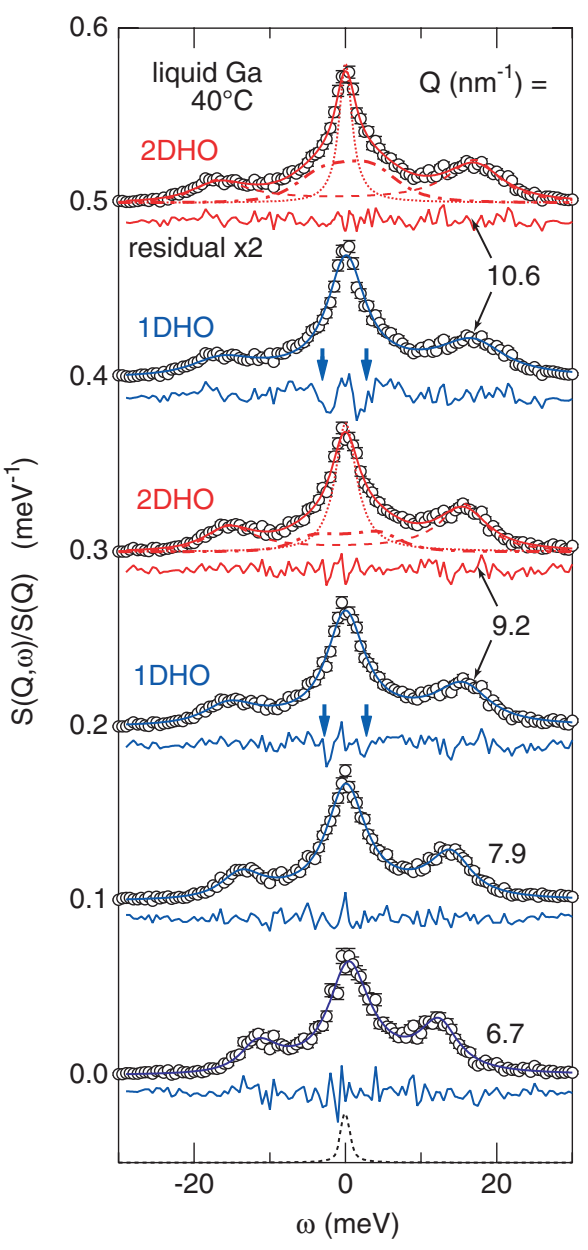

FIG. 1 (color online). $\quad S(Q, \omega) / S(Q)$ at $Q=6.7-10.6 \mathrm{~nm}^{-1}$ (circles with error bars) together with the resolution function (dashed curve at the bottom). Solid curves indicate the best fits and residuals of $1 \mathrm{DHO}$ and $2 \mathrm{DHO}$ models. See the text for details.

in the transverselike contribution curves given by the chain curves, the height and area of the transverselike modes seem to largely decrease with decreasing $Q$. Below $Q \sim$ $8 \mathrm{~nm}^{-1}$, fits using a $1 \mathrm{DHO}$ function were sufficient, with the fit routines giving the transverselike mode intensity nearly zero. Thus, the transverselike excitation modes may either merge into the quasielastic peak or rapidly decrease in intensity.

Figure 2 shows the dispersion relation of the transverselike (open circles) and longitudinal (solid triangles) phonon excitation modes obtained from the fits. The present longitudinal results agree well with previous ones $[9,15,16]$. The velocity of the transverselike mode would be slightly larger than $1050 \mathrm{~m} / \mathrm{s}$, much less than half of the longitudinal sound velocity. If it is the transverse mode, the shear modulus $G$ is obtained to be about $6.5 \mathrm{GPa}$.

Low-energy excitations located between the quasielastic line and the longitudinal acoustic phonon modes were hinted at in the $S(Q, \omega)$ spectra of liquid Ga obtained by an $a b$ initio $\mathrm{MD}$ simulation [17] at $429^{\circ} \mathrm{C}$ and $709^{\circ} \mathrm{C}$. 


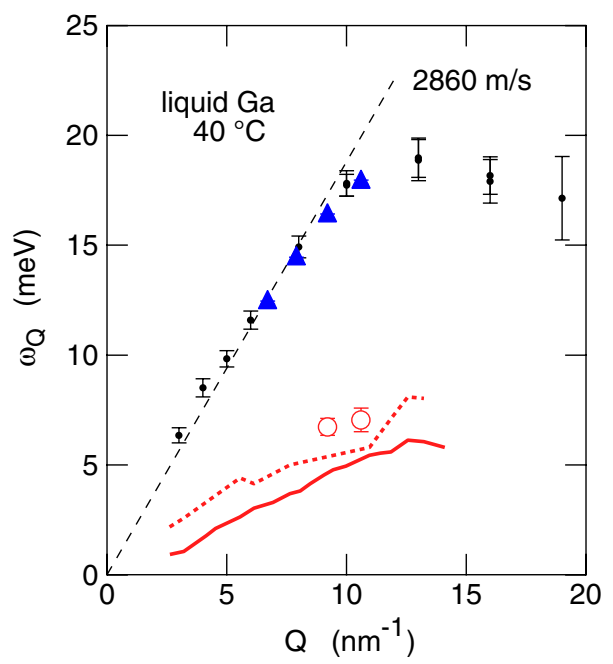

FIG. 2 (color online). Dispersion relation of transverselike (open circles) and longitudinal (solid triangles) modes obtained from the fits. The solid curve gives the peak energies of the transverse current spectra, and the dotted curve is those of the quasitransverse branch seen in the longitudinal current spectra, both obtained by an orbital-free $a b$ initio MD simulation. See the text for details.

Recently, González and González carried out an orbitalfree $a b$ initio $\mathrm{MD}$ simulation on liquid $\mathrm{Ga}$ at $100^{\circ} \mathrm{C}$ and discussed the transverse current correlation functions [18]. This simulation has now been repeated at $40{ }^{\circ} \mathrm{C}$, and the solid curve in Fig. 2 represents the transverse phonon excitation energies $\omega_{\mathrm{Qt}}$ obtained. These theoretical values are slightly smaller than the present experiment values and continue to the lower $Q$ values.

There have been mainly two reasons why the transverse phonon modes were not experimentally studied so far. The first is that they are believed to be difficult to observe at low momentum transfers. Generally, the signal in a typical IXS or INS measurement scales $\vec{u} \cdot \vec{Q}$, where $\vec{u}$ is the displacement of the atom. For the transverse modes in the first Brillouin zone, this product is zero since $\vec{u}$ is always orthogonal to $\vec{Q}$. For crystals, transverse modes are accessible in the second or higher Brillouin zone, with the intensity being proportional to $\vec{u} \cdot(\vec{Q}-2 \pi \vec{H}), \vec{H}$ being a reciprocal-lattice vector chosen along the direction of atomic motion. In the present liquid $\mathrm{Ga}$ case, a first pseudo-Brillouin-zone [19] can be introduced up to $Q \sim$ $12.5 \mathrm{~nm}^{-1}$, half of the first peak positions in $S(Q)$, analogous to the crystal. The periodicity of the dispersion relation at the higher Brillouin zones is, however, broken in disordered materials. The $Q$ range where the transverselike phonon modes are detected in the present experiment is inside the first pseudo-Brillouin-zone but near the zone boundary. Because of the lack of the periodicity in the liquid, however, the boundary of the first and second pseudo-Brillouin-zones may not be strictly divided. Thus, it is possible that the transverse phonon modes can be detected near the pseudo-Brillouin-zone boundary.
The second and the most serious reason is that the transverse phonon modes do not cause directly the density fluctuations, which can be detected by inelastic scattering. Transverse acoustic phonon excitations in liquid water were discussed by Ruocco and Sette [20] using IXS combined with MD simulations. In liquid water, the transverse phonon modes are visible in the IXS spectra in the $Q$ range down to $\sim 3 \mathrm{~nm}^{-1}$, far inside the first pseudo-Brillouinzone, while for solid ice they are seen only beyond $7 \mathrm{~nm}^{-1}$ in the second Brillouin zone. From the analysis of MD simulations, they evaluated the longitudinal and transverse current spectra, both of which show the existence of two excitations, quasilongitudinal and quasitransverse sound branches at $Q>4 \mathrm{~nm}^{-1}$. Because of the lack of the translational invariance, the pure symmetry character of the two modes is rapidly lost at larger $Q$ values, and both of the modes contribute to similar extents to both of the current spectra. Thus, the transverse modes can be detected through the quasitransverse sound branches in the longitudinal current correlation spectrum. In addition, they concluded that the appearance of the propagating transverse dynamics occurs at the same time when the increase of the longitudinal sound velocity takes place from the hydrodynamic to the high-frequency viscoelastic values.

In liquid $\mathrm{Ga}$, the above-mentioned orbital-free ab initio MD simulation also reveals the quasitransverse branch in the longitudinal current spectra as small peaks or shoulders, the energies of which are shown by the dotted curve in Fig. 2 and almost coincide with the peak positions in the transverse current spectra (solid curve). This is clear evidence of the existence of the transverse phonon mode in liquid $\mathrm{Ga}$ measurable by IXS. Thus, it is reasonable to speculate that the detected transverse modes in liquid $\mathrm{Ga}$ would be mainly from the quasitransverse branch of the longitudinal current spectra, causing the density fluctuations. The mixing of the transverse and longitudinal modes in disordered systems would always occur inside the viscoelastic or fast-sound regime for the longitudinal phonons. Whether they are observable or not may depend on the width of the quasielastic line and the excitation energies and widths of the longitudinal mode and transverse mode itself. In the present Ga case, the data at $Q=9.2$ and $10.6 \mathrm{~nm}^{-1}$ may satisfy the good conditions for the observation.

Figure 3(a) shows the widths of the inelastic excitations of the transverse (open circles) and longitudinal (closed triangles) excitations together with the previous longitudinal data (full circles) [9]. The widths of the transverse excitations $\Gamma_{\mathrm{Qt}}$ are about 5-6 meV comparable to its energy $\omega_{\mathrm{Qt}}$ value, 7-8 $\mathrm{meV}$, indicating a highly damped feature, and increases with increasing $Q$ as is the width of the longitudinal phonon excitation width $\Gamma_{\mathrm{Ql}}$. The $\Gamma_{\mathrm{Qt}}$ values are slightly larger than $\Gamma_{\mathrm{Ql}}$, although $\omega_{\mathrm{Qt}}$ is less than half of $\omega_{\mathrm{Ql}}$ at the same $Q$.

Using the $\Gamma_{Q}$ values, the lifetime of these phonon excitations $\tau(Q)$ can be estimated to be proportional to the 


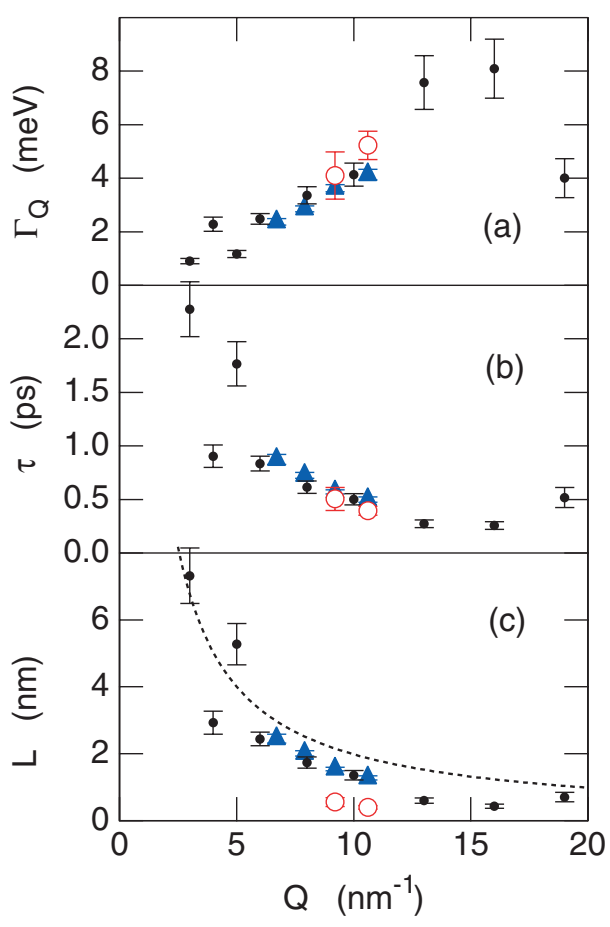

FIG. 3 (color online). (a) The width, (b) lifetime, and (c) propagation length of the transverse (open circles) and longitudinal (closed triangles) excitations together with the previous longitudinal data (full circles) [9]. The dashed curve indicates the Ioffe-Regel criterion [21].

inverse of $\Gamma_{Q}$, i.e., $\tau(Q)=h /\left(2 \hbar \Gamma_{Q}\right)=\pi / \Gamma_{Q}$, where $h$ is the Planck constant and $\hbar=h / 2 \pi$. Figure 3(b) shows the lifetime of the transverse and longitudinal acoustic phonons. The lifetime of the transverse phonons is about $0.5 \mathrm{ps}$, which is almost the same as that of the longitudinal acoustic phonons. Interestingly, this time scale matches that obtained from the linewidth of the quasielastic peak near the $S(Q)$ maximum $\left(0.39 \mathrm{ps}\right.$ at $\left.100^{\circ} \mathrm{C}\right)$ [10]. This time scale was interpreted as the lifetime of short-lived covalent bonds. Such short-living bonds may also indicate the lifetime of the cages formed in liquid Ga instantaneously. Another time scale obtained from the present experiment is the Maxwell relaxation time $\tau_{M}$ [4,6], which can be evaluated from the $G$ value and the shear viscosity. The estimated $\tau_{M}$ is about $0.30 \mathrm{ps,} \mathrm{which} \mathrm{is} \mathrm{again} \mathrm{in} \mathrm{the} \mathrm{same}$ range as the other microscopic values.

The propagating lengths of the phonons $L(Q)$ can be approximated by multiplying the velocity of sound $\omega_{Q} / Q$, expressed as $L=\left(\pi / \Gamma_{Q}\right)\left(\omega_{Q} / Q\right)=\pi \omega_{Q} / Q \Gamma_{Q}$. Figure 3(c) shows the $L(Q)$ values of the transverse (open circles) and longitudinal (closed triangles) acoustic phonons. The propagation length of the transverse excitations $L_{t}$ is about $0.4-0.5 \mathrm{~nm}$. The dashed curve in this figure indicates the Ioffe-Regel criterion $\left(L_{\mathrm{IR}}=2 \pi^{2} / Q\right)$ [21], which is considered as a boundary between the propagating and localized modes. Since the $L_{t}$ values are much shorter than $L_{\mathrm{IR}}$, the transverse mode can be judged as a localized mode. Thus, the $L_{t}$ value may correspond to the size of the cages formed in liquid $\mathrm{Ga}$, where the phonon packets are localized.

In conclusion, we have detected the transverse acoustic excitation modes by IXS in liquid Ga although liquid $\mathrm{Ga}$ is mostly described by a hard-sphere liquid [15]. An orbitalfree $a b$ initio MD simulation clearly supports this finding. From the detailed analysis for the $S(Q, \omega)$ spectra with a good statistic quality, the lifetime of $0.5 \mathrm{ps}$ and the propagating length of $0.4-0.5 \mathrm{~nm}$ can be estimated for the transverse acoustic phonon modes, which may indicate, respectively, the lifetime and size of cages formed instantaneously in liquid Ga.

This work was partially supported by the Promotion and Mutual Aid Corporation for Private Schools of Japan. M. I., Y.K., K.M., and T.I. acknowledge the Ministry of Education, Culture, Sports, Science and Technology of Japan for Grant-in-Aids for Scientific Research. The IXS experiments were performed at the beam line BL35XU in the SPring-8 with the approval of the Japan Synchrotron Radiation Research Institute (Proposal No. 2008A1064).

*Corresponding author. hosokawa@cc.it-hiroshima.ac.jp

[1] A. Polian et al., Europhys. Lett. 57, 375 (2002).

[2] D. Levesque et al., Phys. Rev. A 7, 1690 (1973).

[3] J.-P. Hansen et al., Phys. Rev. A 11, 1025 (1975).

[4] J.P. Boon and S. Yip, Molecular Hydrodynamics (McGraw-Hill, New York, 1980).

[5] U. Balucani and M. Zoppi, Dynamics of the Liquid State (Clarendon Press, Oxford, 1994).

[6] J.-P. Hansen and I. R. McDonald, Theory of Simple Liquids (Elsevier, Amsterdam, 2006), 3rd ed.

[7] F. Shimojo et al., J. Phys. Soc. Jpn. 63, 1821 (1994).

[8] G. Kahl and S. Kambayashi, J. Phys. Condens. Matter 6, 10897 (1994).

[9] S. Hosokawa et al., Physica (Amsterdam) 350B, 262 (2004).

[10] S. Hosokawa et al., J. Phys. Condens. Matter 20, 114107 (2008).

[11] A. Q. R. Baron et al., J. Phys. Chem. Solids 61, 461 (2000).

[12] K. Tamura et al., Rev. Sci. Instrum. 70, 144 (1999).

[13] S. Hosokawa and W.-C. Pilgrim, Rev. Sci. Instrum. 72, 1721 (2001).

[14] B. Fåk and B. Dorner, Institut Laue-Langevin Report No. 92FA008S, 1992.

[15] T. Scopigno et al., Phys. Rev. Lett. 89, 255506 (2002).

[16] L. E. Bove et al., Phys. Rev. B 71, 014207 (2005).

[17] J. M. Holender et al., Phys. Rev. B 52, 967 (1995).

[18] L. E. González and D. J. González, Phys. Rev. B 77, 064202 (2008).

[19] T. Scopigno et al., Phys. Rev. B 64, 012301 (2001).

[20] G. Ruocco and F. Sette, J. Phys. Condens. Matter 11, R259 (1999).

[21] A. F. Ioffe and A. R. Regel, Prog. Semicond. 4, 237 (1960). 